\title{
Crystal structure analysis and conformational epitope mutation of triosephosphate isomerase, a mud crab allergen
}

Fei Xia ${ }^{1}$, Meng-Si Li ${ }^{1}$, Qing-Mei Liu ${ }^{1}$, Meng Liu ${ }^{1}$, Yang Yang ${ }^{1}$, Min-Jie Cao ${ }^{1}$, Gui-Xia Chen ${ }^{2}$, Tengchuan Jin ${ }^{3 *}$ and Guang-Ming Liu ${ }^{1 *}$

${ }^{1}$ College of Food and Biological Engineering, Xiamen Key Laboratory of Marine Functional Food, Fujian Provincial Engineering Technology Research Center of Marine Functional Food, Fujian Collaborative Innovation Center for Exploitation and Utilization of Marine Biological Resources, Jimei University, Xiamen, Fujian, China

${ }^{2}$ Women and Children's Hospital Affiliated to Xiamen University, Xiamen, Fujian 361003,

China

${ }^{3}$ Hefei National Laboratory for Physical Sciences at Microscale, CAS Key Laboratory of Innate Immunity and Chronic Disease, Division of Life Sciences and Medicine, University of Science \& Technology of China, Hefei, Anhui, China

Running Title: Structure analysis and conformational epitopes mutation of Scy $\mathrm{p} 8$.

\section{*Corresponding authors}

Tengchuan Jin, School of Life Sciences, University of Science \& Technology of China, Hefei 230027 Anhui, China

Email: jint@ustc.edu.cn

Guang-Ming Liu, College of Food and Biological Engineering, Jimei University, Xiamen 361021 Fujian, China

Email: gmliu@jmu.edu.cn 


\section{Online Supplementary Material}

\section{Supplementary Methods}

\section{Preparation of rabbit anti-crab TIM polyclonal antibodies}

The specific polyclonal antibody was prepared by subcutaneously injecting of the recombinant Scylla paramamosain wtTIM in an adult female New Zealand white rabbit. The rabbit anti-crab TIM polyclonal antibody titer was measured by a dot blot analysis, following the method of Liu et $\mathrm{al}^{1}$. The specific polyclonal antibody (1:5000 dilution) was then recruited as the primary antibody for the cross-reactivity Western blot. 


\section{Supplementary Figure Legends}

FIGURE S1. Determination of the rabbit anti-crab TIM polyclonal antibody titer.

Dot blot analysis of the rabbit anti-crab TIM polyclonal antibody titers, 1:100 - 1:2 $\times$ $10^{4}$ is the polyclonal antibody dilution.

FIGURE S2. Thermal denaturation analysis of mutant TIMs by CD spectroscopy.

A - E. Determination of the thermal denaturation temperature values of wtTIM, W168A, T172A, Q180A, and K237A, respectively.

FIGURE S3. Effect of wtTIM and mutant TIMs on the expression of CD63 and CD203c in basophils.

A. Patients with AC $(n=6)$ were treated with wtTIM; the blood $(n=2)$ of NA was used as the negative control.

B and C. Patients P2, P4, P7, P8, P10, P11 were treated with W168A, T172A, Q180A, and K237A, respectively. PBS is shown in gray, orange for wtTIM, and the lines indicate mutant TIMs.

FIGURE S4. The electrostatic surface potential of mutants T172A and Q180A.

A. The electrostatic surface potential of wtTIM, T172A and Q180A. Red and blue are negative and positive charge, respectively.

B. The electrostatic surface potential of T172A at another Angle. 
Table S1. Oligonucleotide primers used in PCR.

\begin{tabular}{|c|c|}
\hline Primers & Oligonucleotide sequence (5'-3') \\
\hline wtTIM-F & 5'-CATATGATGGCCAACCAGAGGAAAT-3' \\
\hline wtTIM-R & 5'-GTCGACTTAAGCACGGGCATTAATAATT-3' \\
\hline W168A-F & 5'-AGTA $\underline{\boldsymbol{G} \boldsymbol{C} \boldsymbol{A}}$ GCTATTGGAGCGGAAAG-3' \\
\hline W168A-R & 5'-TAGC $\underline{\boldsymbol{T} G \boldsymbol{C}}$ TACTGGTTCATAAGCAAT-3' \\
\hline T172A-F & 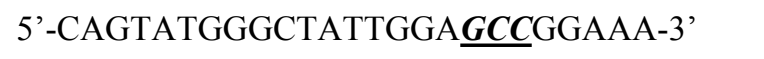 \\
\hline T172A-R & 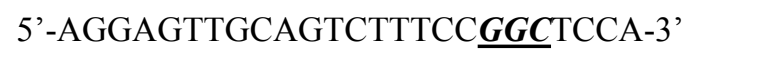 \\
\hline Q180A-F & 5'-GAAAGACTGCAACTCCTGAA $\underline{\boldsymbol{G} \boldsymbol{C A}} \mathbf{G C - 3}$ ' \\
\hline Q180A-R & 5'-GGACATCTTGGGC $\underline{\boldsymbol{T G C}}$ TTCAGGAGT-3' \\
\hline K237A-F & 5'-TTGTGGGAGGTGCTTCCCTC $\underline{\boldsymbol{G C A C C}-3}$ ' \\
\hline K237A-R & 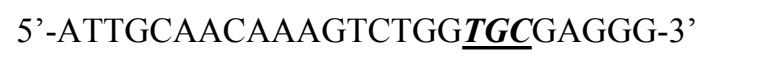 \\
\hline
\end{tabular}

The nucleotide changes are underlined; $\boldsymbol{C A T A T G}$ and $\boldsymbol{G C T G A C}$ restriction sites for Nde I and Sal I enzymes, respectively. 
FIGURE S1

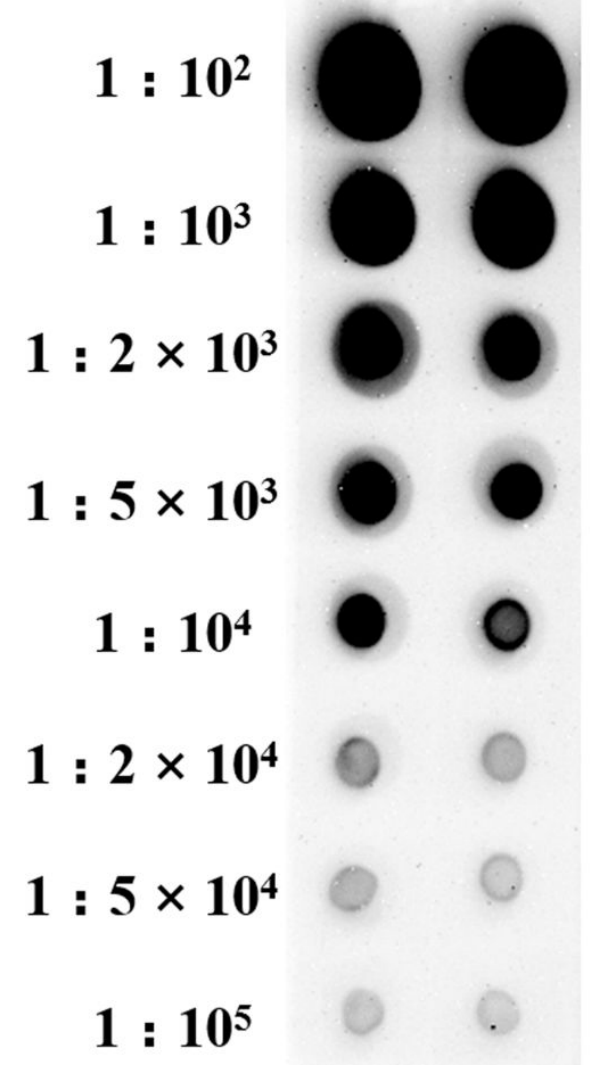




\section{FIGURE S2}

A

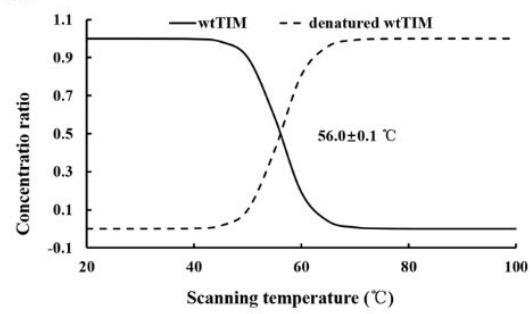

B

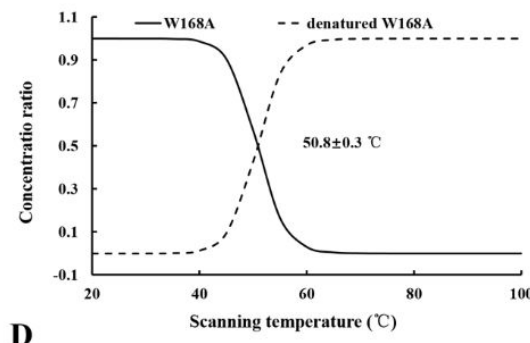

C
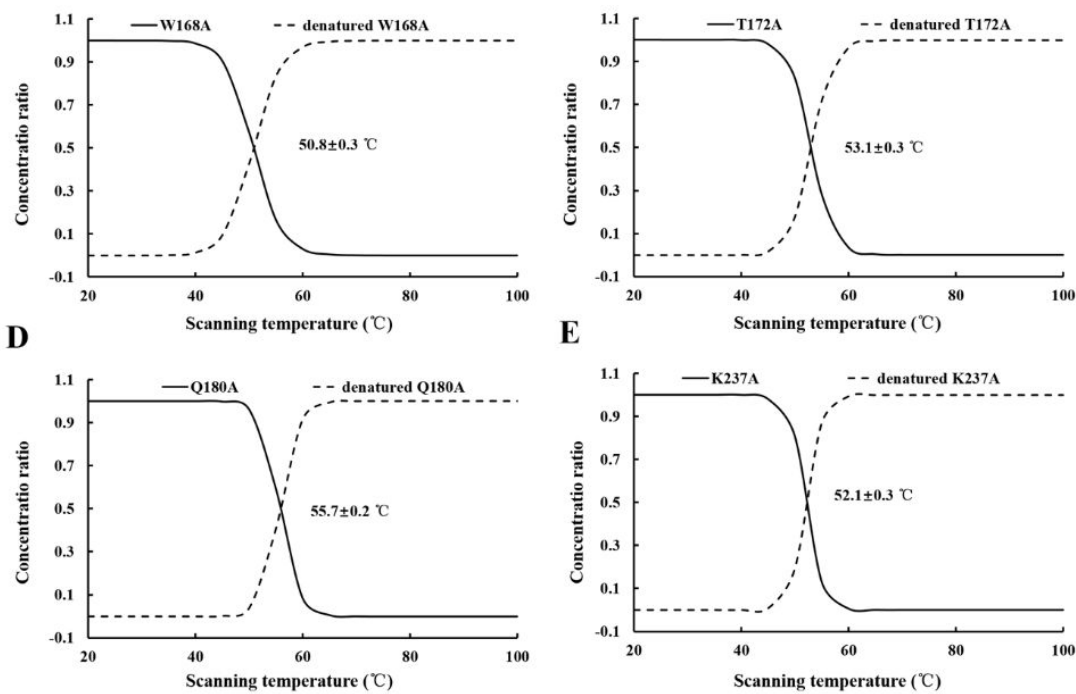
FIGURE S3

A

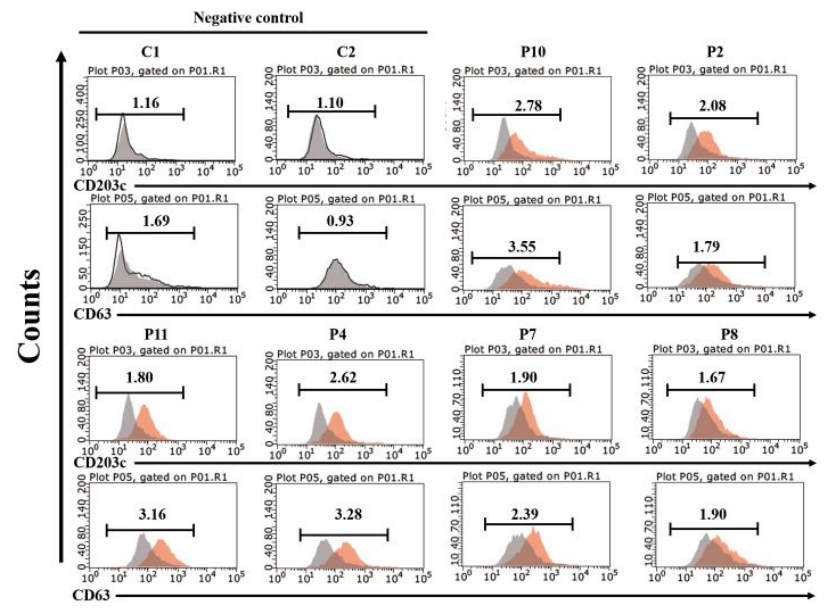

B

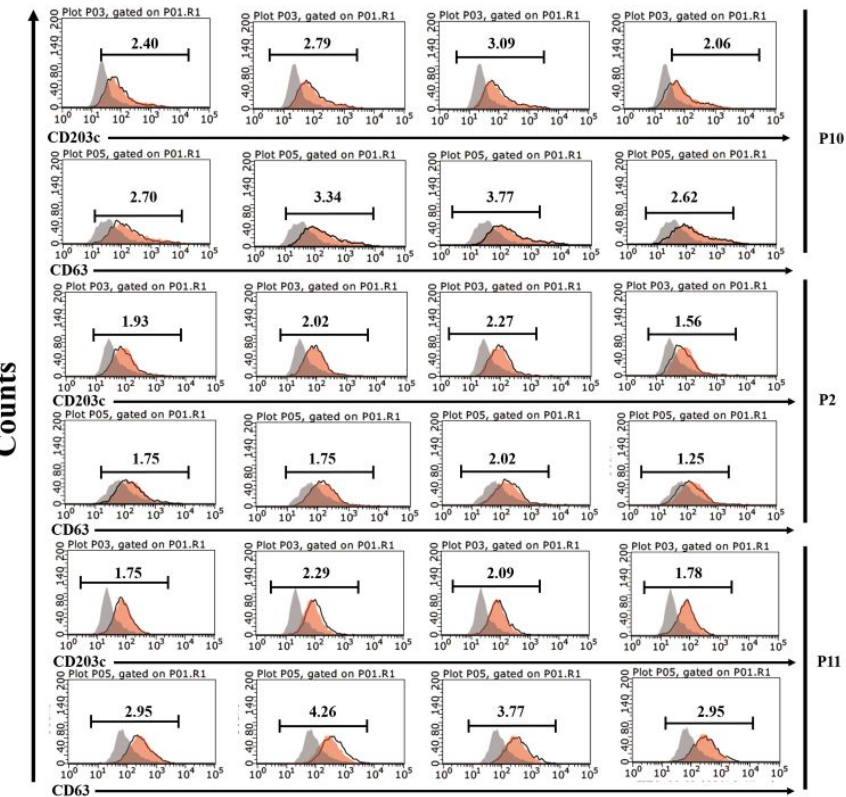

C

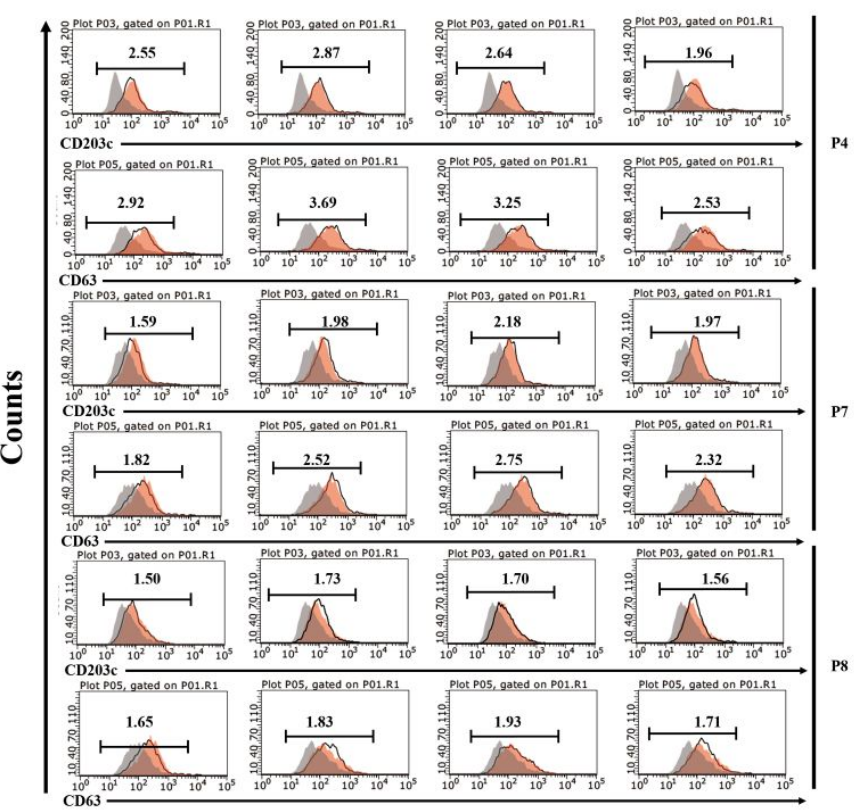


FIGURE S4
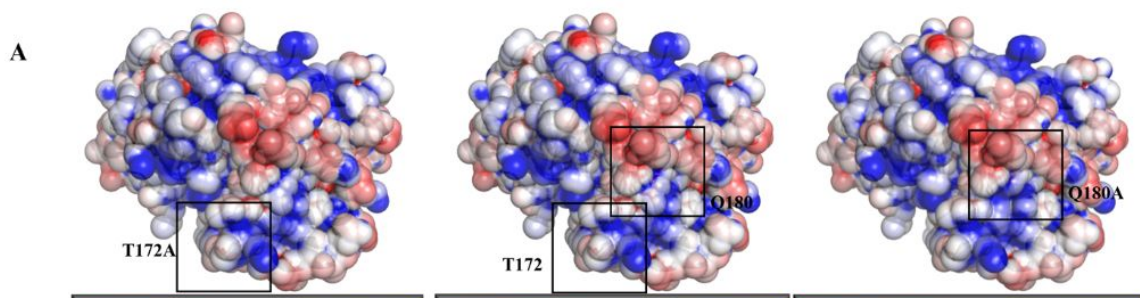

B

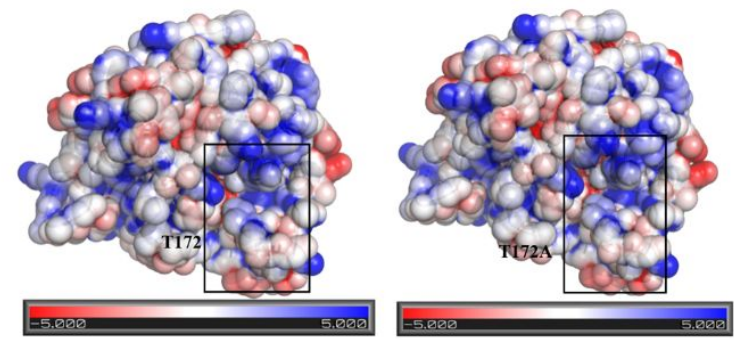




\section{References}

1. Liu, Y. Y.; Cao, M. J.; Zhang, M. L.; Hu, J. W.; Zhang, Y. X.; Zhang, L. J.; Liu, G. M. Purification, characterization and immunoreactivity of $\beta^{\prime}$-component, a major allergen from the roe of large yellow croaker (Pseudosciaena crocea). Food Chem. Toxicol. 2014, 72, 111-121. 